\title{
Inflight-Measurements of Aircraft Undercarriage Vibration during Deployment
}

\author{
Dr.-Ing. Jan Schwochow ${ }^{1}$, Julian Sinske ${ }^{1}$, Ralf Buchbach ${ }^{1}$ \\ ${ }^{1}$ DLR Institute of Aeroelasticity, Bunsenstrasse 10, 37073 Göttingen, Germany, \\ jan.schwochow@dlr.de
}

\begin{abstract}
:
Within the EU funded Project AFLoNext (http://www.aflonext.eu/) flow-induced vibrations of aircraft components are under investigation. Aerodynamic or structural devices are designed to mitigate the vibration response levels especially of the deployed landing gear doors. The nose landing gear is emitting vorticity which impacts the downstream located main landing doors. To verify the results of unsteady complex flow simulations of the undercarriage conducted by different project partners, flight tests shall provide a database for calibration of numerical investigations and verify the results. Different types of sensors were installed on the undercarriage of the Advanced Technology Research Aircraft operated by the German Aerospace Center DLR, comprising accelerometers, strain gauges, travel and pressure sensors. To get detailed knowledge of the dynamic interaction of pressure loads and structural response on the nose and main landing gear doors, the simultaneous data acquisition of all signals at high sample rates up to $2 \mathrm{kHz}$ was mandatory during deployment. In addition, monitoring of critical response levels was essential to stay in defined limits during flight. The presentation will give an overview of the selected Flight Test Instrumentation and the preparation of the test aircraft including the documentation and substantiation to get the flight approval.
\end{abstract}

Key words: AFLoNext, ATRA, landing gear, distributed DAQ, flight vibration test, flight test instrumentation.

$\begin{array}{ll}\begin{array}{l}\text { Glossary } \\ \text { A/D }\end{array} & \begin{array}{l}\text { Analog / Digital converter } \\ \text { AFLoNext }\end{array} \\ & \begin{array}{l}\text { Active Flow Loads \& Noise } \\ \text { Control on Next Generation } \\ \text { Wing (EU-Project) }\end{array} \\ \text { ATRA } & \begin{array}{l}\text { Advanced Technology Research } \\ \text { Aircraft (AIRBUS A320) }\end{array} \\ \text { CFD } & \text { Computational Fluid Dynamics } \\ \text { DAQ } & \text { Data Acquisition } \\ \text { DLR } & \text { German Aerospace Center } \\ \text { DO } & \text { Design Organisation } \\ \text { EASA } & \text { European Aviation Safety } \\ \text { EMA } & \text { Agency } \\ \text { EMC } & \text { Experimental Modal Analysis } \\ \text { F/T } & \text { Flight Test } \\ \text { FTI } & \text { Flight Test Instrumentation }\end{array}$

$\begin{array}{ll}\text { FVT } & \text { Flight Vibration Test } \\ \text { GVT } & \text { Ground Vibration Test } \\ \text { MLG } & \text { Main Landing Gear } \\ \text { NLG } & \text { Nose Landing Gear } \\ \text { OMA } & \text { Operational Modal Analysis } \\ \text { PtF } & \text { Permit to Fly }\end{array}$

\section{Introduction}

The vibrations on the aircraft undercarriage triggered by unsteady aerodynamics forces are relevant for the design of airframe components. Being generated by unsteady flow around itself or excited by vortices shed from other upstream components, e.g. the nose landing gear (NLG), these vibrations are often present on movable surfaces in the undercarriage area like the main landing gear doors (MLGD) with a potentially significant impact on airframe noise and component fatigue, even though they are open only for a short sequence while the MLGs are in transit. During the years, semi-empirical methods were used to anticipate to flight tests. 
Nowadays, high-precision computational fluid dynamics (CFD) simulation tools are becoming available that are capable for predicting unsteady aerodynamic phenomena as well as the dynamic response of the structure prior to $F / T$. An example result for visibility of the vorticity from [1] is shown in Figure 1, which appears, when the LG is in transit (see Figure 2) These capabilities allow the thoroughly investigation of the root causes of transport aircraft undercarriage component vibrations and on the other hand the design of small aerodynamic devices, which might impact the flow vorticity to mitigate the excitation levels of the structural response and prevent damages. In best case these devices can be retrofitted to an existing aircraft fleet. Details can be found in [1] and [2].

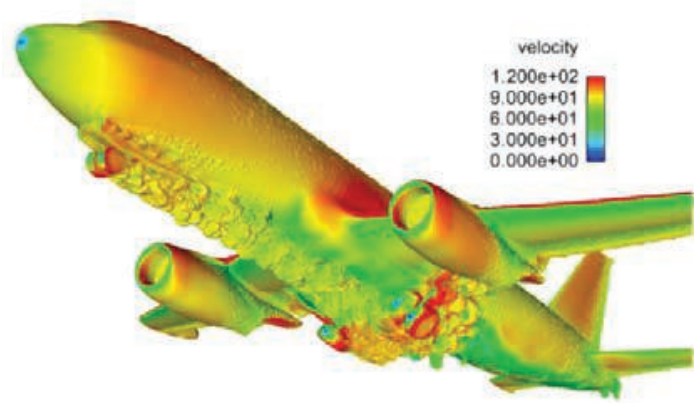

Figure 1. CFD-simulation of undercarriage vorticity from [2].

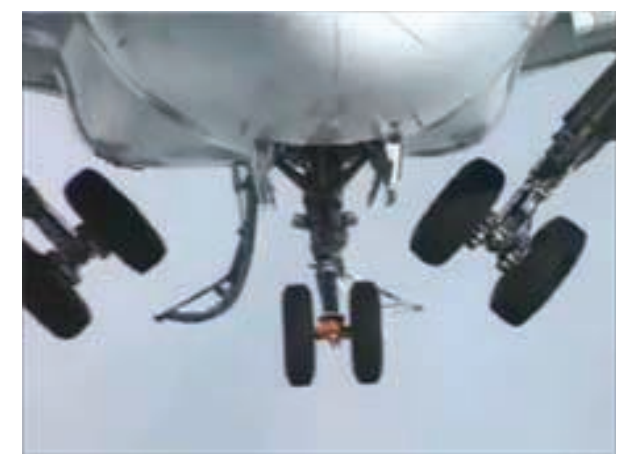

Figure 2. Landing gear in transit.

The numerical simulation of the vibration level of the undercarriage is associated with uncertainties because of detached flow, vortex shedding and buffet. The unsteady aerodynamic forces of the disturbed flow are exciting the extended gears with opened doors. Again, the oscillating aircraft components influence the kind of aerodynamic characteristics, which results in a coupled aerodynamic and structural interaction. These new multi-disciplinary simulation tools are still in the proving phase, which needs experimental $F / T$ results to check their prediction capabilities.
Consequently, the research activities within the AFLoNext project funded by the European Union [3] comprises instrumented $F / T$ with the Advanced Technology Research Aircraft (ATRA) operated by the Flight Experiments Department of the German Aerospace Center (DLR) [4]. The ATRA is originally a commercial A320 (see Figure 3), which was reconfigured to a research platform equipped with special data acquisition systems to allow comprehensive flight testing for customers.

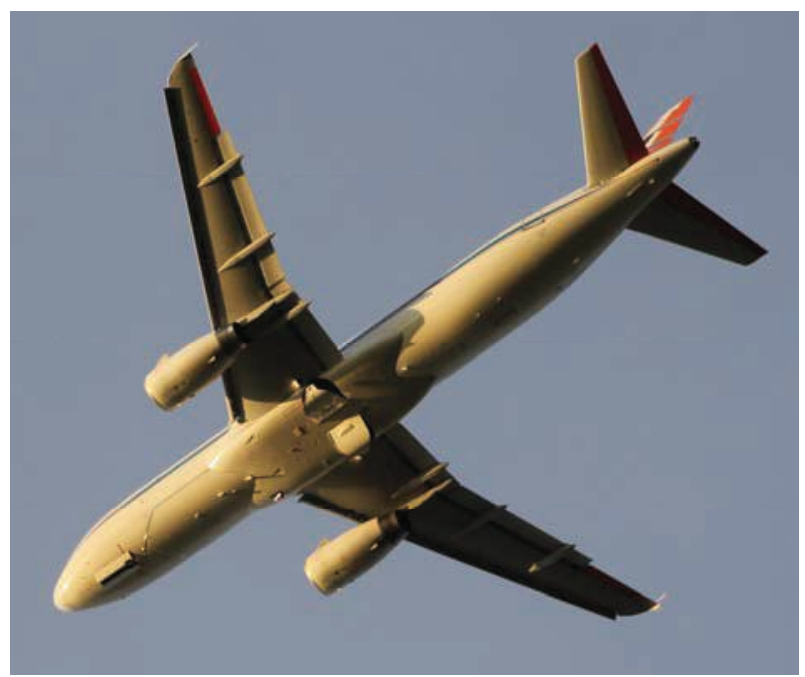

Figure 3. A320 ATRA - Advanced Technology Research Aircraft, shortly after take-off.

To support the investigations on the undercarriage, a small rugged and customizable data acquisition system (DAQ) was installed outside of the aircraft cabin in the MLG bay containing the signal conditioning and $A / D$-converters of different type of sensors:

- accelerometers,

- $\quad$ pressure transducer,

- $\quad$ strain gauges, and

- travel sensors.

Furthermore, ground tests on landing gear doors were performed to analyze the dynamic characteristics of the considered door components without aerodynamic disturbances occurring in flight. The landing gears with deployed doors comprise numerous movable cranks and hinges and hydraulics (see Figure 2), which is expected to cause nonlinear vibration characteristics due to friction and freeplay within the built-in kinematics. To separate between structural phenomena and aerodynamic excitation effects the structural dynamic properties must be identified during a ground vibration test (GVT), where the aircraft is on jacks. By application of experimental modal analysis (EMA), the dominant vibration 
mode shapes with natural frequencies can be identified. These modal results will be exploited for calibration and verification of the structural modelling which is applied in numerical simulation.

Besides the clean MLGD configuration, Vortex Generators (VG) on the outer surface of the doors (see Figure 4) and aerodynamic deflectors on the inner leading edge (see Figure 5) were installed and tested in flight to control the flow with respect to vibration mitigation.

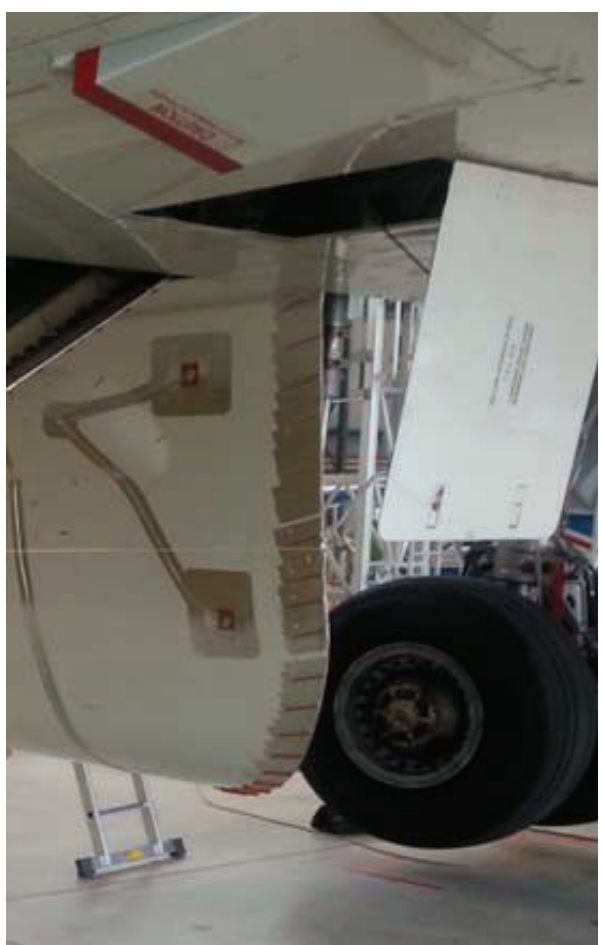

Figure 4. Vortex generators on the MLGD leading edge.

\section{Requirements for Flight Test Instrumentation}

To fully understand the aircraft undercarriage phenomena the measurement of the current position of NLG as vortex shedding generator deflection angle of MLG doors, unsteady aerodynamic loads on the MLG doors, the vibration level response were requested by the simulation experts. Supplementary, a camera with high frame rate shall record the MLG and doors in transit.

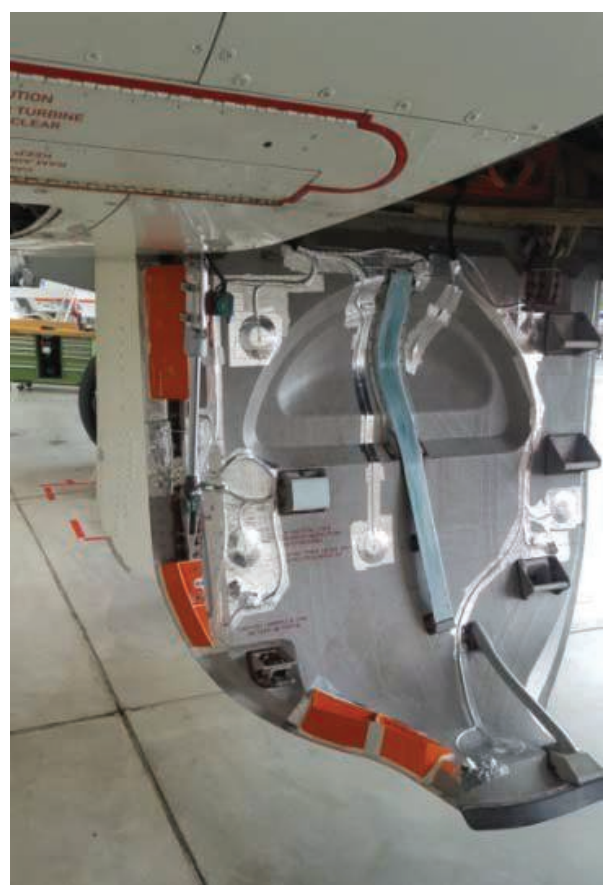

Figure 5. Aerodynamic deflectors at leading edge of MLGD.

The final Flight Test Instrumentation comprises the following installations:

- $\quad$ position measurement of the NLG leg via travel sensors (string potentiometer) MIDORI CPP45-50 to measure piston stroke at the NLG actuator (see Figure 6),

- $\quad$ position measurement of both MLG doors via string potentiometer CPP4550 at actuator,

- total load measurement of the applied load on each $L G$ door via strain gauges at control rods of NLG + MLG doors (see Figure 7),

- vibration level measurement via accelerometers PCB-353B17: 3x triaxial, $1 x$ bi-axial, 1x uni-axial sensors on each MLG door (see Figure 8) and $4 x$ uni-axial on each NLG door (see Figure 9),

- $\quad$ aerodynamic load measurement: via pressure transducers KULITE GG835 on in-/outboard surface of left-hand MLG door (see Figure 8).

All sensors were glued to the aircraft surface with $3 \mathrm{M}$ Scotch-Weld two component epoxy adhesive DP490 [7], which is gap filling and durable down to low temperatures $\left(-55^{\circ} \mathrm{C}\right)$. All outboard cables were glued to the aircraft surface with aluminium foil tape 3M 425 [8]. 


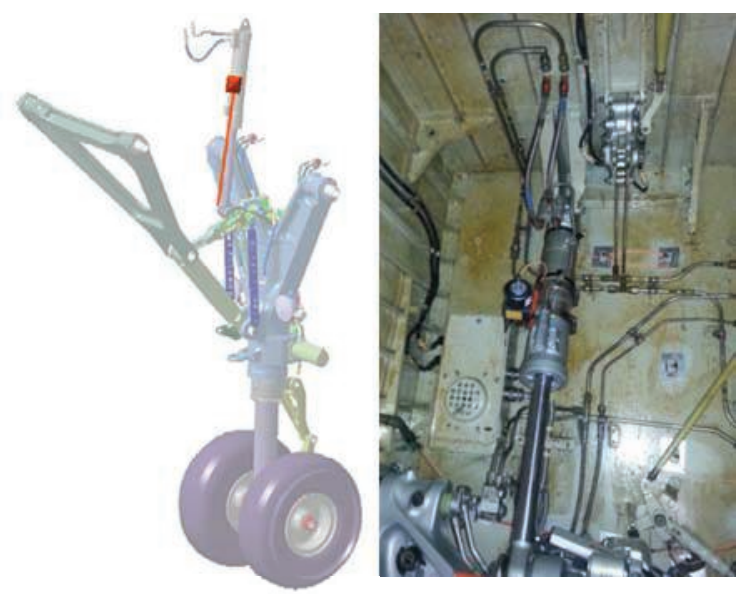

Figure 6. Travel sensor at NLG actuator.

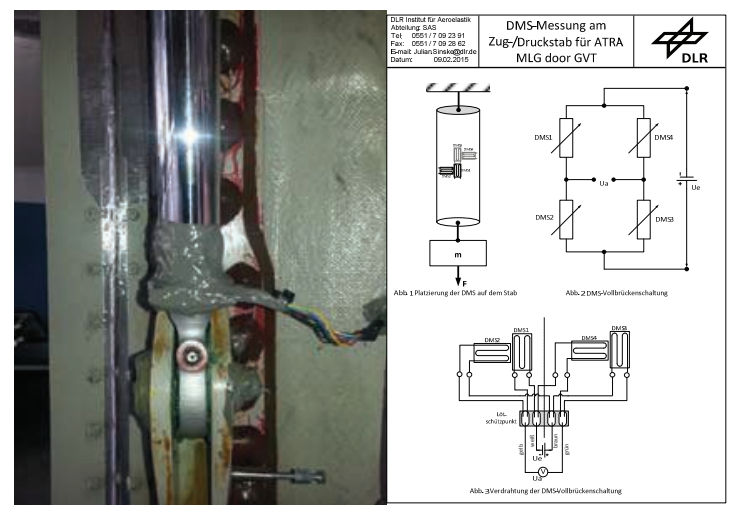

Figure 7. Strain gauge at MLG actuator.

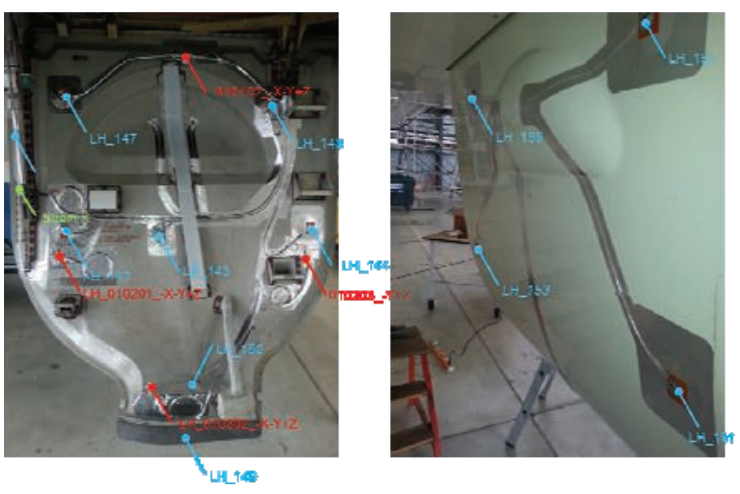

Figure 8. Accelerometers (red) and pressure transducer (blue) on left-hand MLG door.

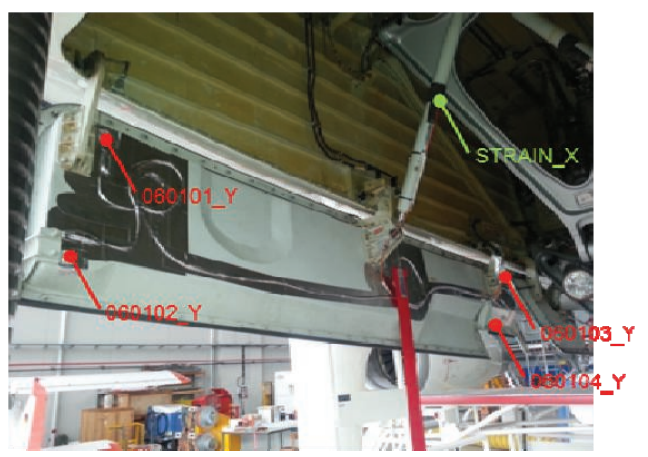

Figure 9. Accelerometers and strain gauge at righthand NLG door.

\section{Data Acquisition System}

The signals of all different types of sensors must be acquired and logged simultaneously to hard disc to enable the observation of the correlations between the aerodynamic vortex excitation and the acceleration response of the doors. Furthermore, the functionality of onlinemonitoring of the acceleration response was mandatory to make sure that certain levels are not exceeded during flight.

The available basic DAQ-system in the ATRA has capabilities to record a number of data signals. Since it is installed in the cabin, such a configuration would mean, that all wiring must be routed from outside to the pressurized interior cabin. More than 100 wires would pass a sealed interface located in the fuselage bottom in the MLG bay.

To avoid such an elaborate installation it was searched for an alternative, where the DAQsystem with signal conditioning and A/Dconverters is installed outside of the cabin in the MLG bay. Using such a distributed setup, just the power supply and the network connection need to be routed into the cabin, where the system is controlled via a commercial mobile PC, which records the data to hard disk. According to airworthiness specifications simplified verification can be applied for attachment of external masses in fuselage area if the weight limit is below $10 \mathrm{~kg}$. The mounting of such device must bear a load factor of $10 \mathrm{~g}$, which results to $1 \mathrm{kN}$ limit load.

The distributed setup of the measurement units inside and outside the pressurized cabin required an especially robust system. The system had to work reliably at low temperatures and low air pressure in high altitudes. The selected $\mathrm{NI}$ CDAQ measuring system, which is very compact and allows online access to the time data, is specified by the manufacturer only up to an altitude of $5000 \mathrm{~m}$ and a temperature down to $-40^{\circ} \mathrm{C}$. This limited specification required a climatic chamber test in which the requirements of a flight profile were simulated, as it would occur during the targeted flight tests. During this climatic chamber test, the measuring system together with the sensors was exposed to a pressure of less than 250 mbar, which simulated an altitude of approx. $11,500 \mathrm{~m}$ and a temperature of below $-50^{\circ} \mathrm{C}$. The measurement system worked perfectly during this $3 \mathrm{~h}$ test and showed no drifts in the measured signals, no errors or any failure.

The complete setup of the distributed measuring system (see Figure 10) consists of two individual National Instruments chassis of type NI-cDAQ-9188XT [5]. Each contains eight 
adaptable signal conditioning modules to support the need of the different sensor types. Both were covered by a rugged protective aluminum case box, which was attached on the keel beam of the aircraft in the MLG bay (see Figure 10). The total weight of this LoadDAQbox is $9.5 \mathrm{~kg}$, while the voltage power supply is $28 \mathrm{~V}$. This outboard installation permits considerable shortening of the analog signal routing, which improves the signal to noise ratio and reduces the electromagnetic susceptibility from radio emission of the different communication antennas. A further request for the flight test qualification was a check of electromagnetic compatibility as part of an EMC test in an EMC chamber and later in the installed state on the entire aircraft. These tests intended to exclude the measurement electronics from influencing important components of the aircraft such as radio communication. In several former flight tests the $\mathrm{NI}-\mathrm{cDAQ}-9188 \mathrm{XT}$ has proved oneself as robust and reliable system [9].

The architecture of the DAQ-network consists of a central DAQ-PC, which is connected to the local aircraft network and records the data from the two $\mathrm{NI-cDAQ}$ chassis with sample rate of $2 \mathrm{kHz}$ and at the same time the flight condition parameters comprising flight speed, Mach number, flight altitude, static pressure, angle of attack, yaw angle and other made available by the basic aircraft DAQ-system at sample rate of $10 \mathrm{~Hz}$.

This main DAQ-PC distributes these data in the local network, so that further online evaluation and processing can be performed with additional PCs connected to the local network. In Figure 11 the architecture is summarized. The $\mathrm{NI}$ CDAQ-chassis are filled with the following conditioned I/O modules:

- 2x NI 9469 - synchronization of chassis,

- $8 x$ NI 9234 - acceleration with IEPEsignal conditioning $+/-5 \mathrm{~V}, 4$ channels per module, BNC

- 3x NI 9234 - pressure transducer, +/$5 \mathrm{~V}$, IEPE disabled, 4 channels per module, BNC,

- $2 x \mathrm{NI} 9237$ - +/-25mV/V full bridge analog input with $10 \mathrm{~V}$ internal excitation and remote sensing, 4 channels per module,

- 1 x NI $9239- \pm 10 \mathrm{~V}$ simultaneous analog input for travel sensor, 4 channels per module, BNC.

Figure 12 shows the electrical block diagram of the $\mathrm{FTI}$, where the three cable-line connection from MLG bay into cabin is indicated. The connection between MLG and NLG bay comprises several sensor cables, as shown in Figure 13.

\section{Data Acquisition Software}

The software to control the measurement system and to acquire the signals is developed with NI LabVIEW 2016. Both NI-CDAQ chassis must be synchronized and all channels must be pre-defined with particular signal conditioning settings. Further, an infrastructure of networkstreams is installed to continuously feed several analysis-computers connected to the network with live-data.

The basic data acquisition system of the aircraft supplies the current flight parameters via an additional network stream. These data are collected and stored synchronously with the measurements on the undercarriage.

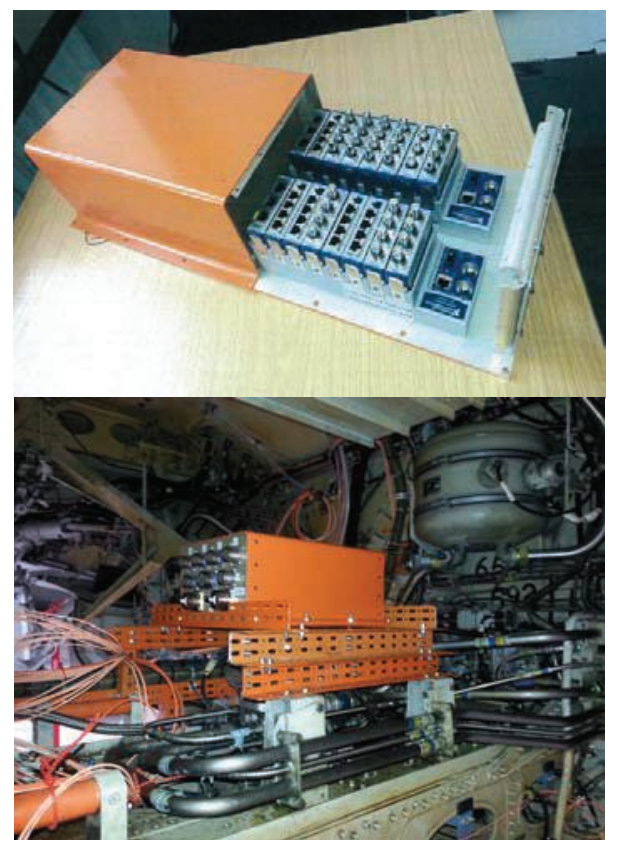

Figure 10. LoadDAQ box installed on the aircraft keel beam in the MLG bay.

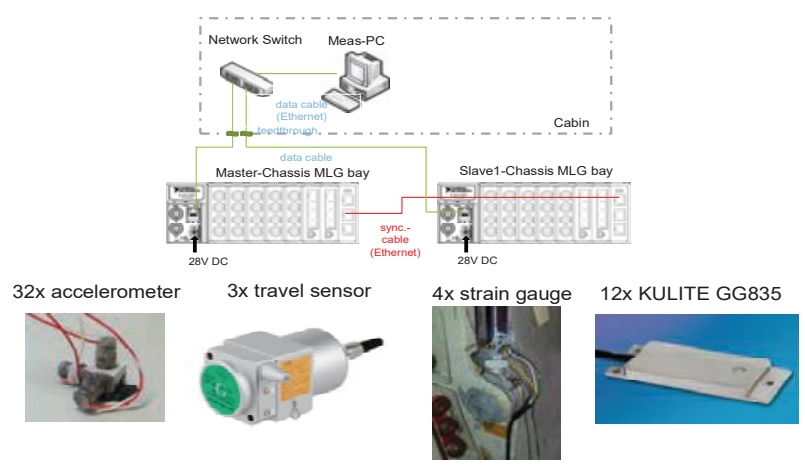

Figure 11. LoadDAQ-System with different sensor types. 


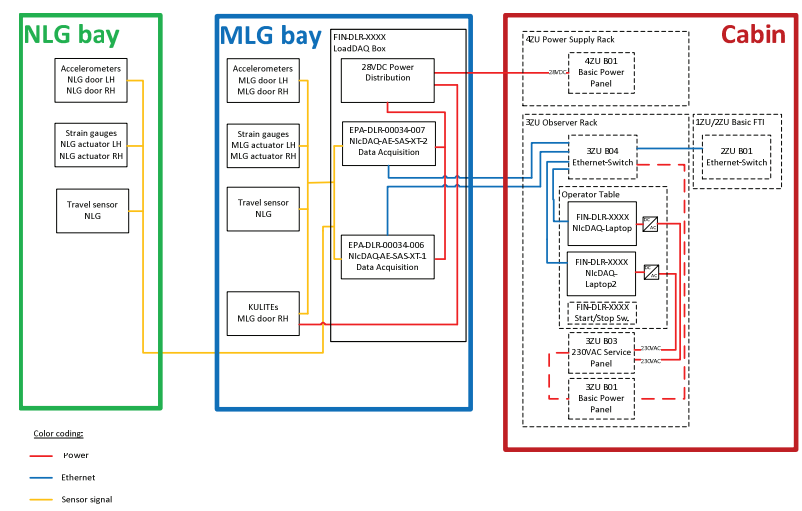

Figure 12. Electrical block diagram of distributed $D A Q$.

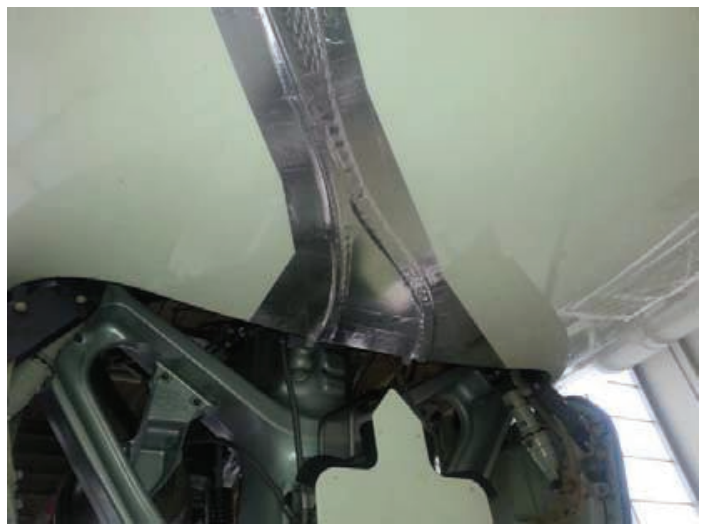

Figure 13. Cable connection to NLG bay.

\section{Qualification for Flight Test}

In order to get the permit to fly (Ptf) for the aircraft equipped with FTI several documentation and substantiation reports must be generated and approved if the installation is compliant with the aircraft certification specifications CS-25 published by the European Aviation Safety Agency (EASA) [5]. This approval step guarantees a safe performance of the test flight. The German Aerospace Center (DLR) operates a Design Organisation (DO) to conduct research activities for users from DLR institutes, external research establishments, industry and government authorities / agencies. That comprises the development, type investigation and supplemental type certification of interior and exterior test equipment / attachments or modifications in compliance with applicable airworthiness requirements.

The DO itself grants the supplemental type certification for minor changes within the legally allocated area. Therefore it administrates governmental tasks and acts as part of the administration authority of the Federal Republic of Germany.
The capabilities of the DO are the indispensable prerequisite for the efficient operation of the DLR aircraft fleet in all areas of flight environmental research. In addition to the scientific airplane researches, the main focus lies on the airplanes as a flying laboratory. The application area covers atmospheric research, earth observation and traffic research.

In case of the FTI installation on undercarriage the staff generated compliance reports for the following technical disciplines:

- Electrical substantiation,

- Structural substantiation, and

- Aeroelastic substantiation.

In so-called Special Instructions (SI) the installation procedure is written down step by step, so that the technical staff can follow the instructions and guarantee a qualified installation of the test equipment.

\section{Flight Test Results}

In Figure 14 flight test results are plotted for the deployment sequence of the landing gear at maximum speed of $250 \mathrm{kts}$. The first diagram shows the deflection angles of NLG leg and lefthand MLG door and the total load applied on the door (blue curve) and measured via the strain gauge glued on the actuator rod (see Figure 7). The second row contains the accelerations and the third row shows the measured pressures (see Figure 8).

The course of the overall load measured by the actuator strain gauge needs some explanation. In the beginning, when the door is closed, the actuator pulls the door against the locking device. This avoids the dropping of the door caused by its own weight, when the locker is suddenly released. At this moment the pulling force is reduced to almost zero and the door opens first by its weight and later when there is gap between door and fuselage bottom by the surrounded aerodynamic forces with tendency to open. When the door is fully deployed sequence $C-$, the MLG starts to swing and passes the door. Due to the expulsion of the landing gear wheels additional aerodynamic loading is generated, which pulls the door into the stops. At half deflection angle the force is at its maximum - sequence $\mathrm{E}-$, which represents the static limit load for the door design. Dynamic loads caused by the vibration occur on top of this static condition. So, when the vibration level can be reduced the fatigue loads are decreased as well. This is the reason, why different aerodynamic and structural devices for vibration mitigation are under investigation in 
the AFLoNext research project. At the end sequence $G$ - the door is closed and the actuator pulls against the locker again.

The impact of the NLG as vortex shedding generator can be observed from the comparison of the acceleration level at sequence $\mathrm{C}$, when all doors are open but the NLG is still in the bay, and at the sequence $F$, when the NLG is down. The same interrelation is observed in the retraction sequence between $B$ and $E$ in Figure 15. Here, the maximum speed is limited to $220 \mathrm{kts}$ due to the flight manual, which results in slightly lower maximum total loads in the actuator.

The flight test matrix contains several test points at low and high flight speeds and different angles of attack and yaw angles.

These test points were repeated for the checkout flights of the different aerodynamic and structural devices installed on the MLG doors to mitigate the loading and the vibration response.

Finally, a database was filled with experimental flight test results, which serves as the validation reference for the new high-precision CFDsimulation tools to predict unsteady aerodynamic phenomena as well as the dynamic response of the aircraft undercarriage.

\section{Conclusions}

The paper presents the preparation, execution and research scope of the AFLoNext flight test campaign dedicated to vibration measurements on the undercarriage of DLR's research aircraft A320 ATRA.

The suitability of the relatively inexpensive NI$\mathrm{CDAQ}$ measuring system for airborne test conditions has been successfully demonstrated during the flight test campaign under real conditions installed in the MLG bay of the ATRA, where the measuring system worked without any failure over the entire duration. The $\mathrm{NI-CDAQ}$ system permits customizable signal conditioning for several types of sensor comprising strain gauges, accelerometers, potentiometers and pressure transduce installed on all landing gear doors. The measurement signals can be acquired simultaneously at high sample rates.

The experimental flight test results will serve as a validation database for the upcoming new prediction numerical methods to simulate the dynamics of the overall aircraft undercarriage.

\section{Acknowledgments}

The authors would like to thank all partners involved in the AFLoNext Project and the ATRA Team of the DLR Flight Experiments Department in Braunschweig, Germany. Furthermore, the funding of the AFLoNext flight tests by the European Union within FP7 is gratefully acknowledged.

\section{References}

[1] Shia-Hui Peng, Adam Jirasek, Mats Dalenbring, and Peter Eliasson. "Aerodynamic Excitation on MLG Door Exposed to Vortices Emanating from NLG of an Aircraft Model", 34th AIAA Applied Aerodynamics Conference, AIAA AVIATION Forum, (AIAA 2016-4043) https://doi.org/10.2514/6.2016-4043

[2] Maximilian M. Tomac, Arthur W. Rizzi, Dominique Charbonier, Jan B. Vos, Adam Jirásek, Shia-Hui Peng, Andreas Winkler, Alexander Allen, G. Wissocq, Guillaume Puigt, Julien Dandois, and Ramon Abarca-Lopez. "Unsteady Aero-Loads from Vortices Shed on A320 Landing Gear Door: CFD compared to flight tests", 54th AIAA Aerospace Sciences Meeting, AIAA SciTech Forum, (AIAA 20160803) https://doi.org/10.2514/6.2016-0803

[3] www.aflonext.eu

[4] http://www.dlr.de/fb/en/

[5] http://www.ni.com/dataacquisition/compactdaq/

[6] CS-25, https://www.easa.europa.eu/documentlibrary/certification-specifications

[7] http://multimedia.3m.com/mws/media/827900/ dp490-scotch-weld-tm-adhesive.pdf

[8] https://www.3m.com/3M/en US/companyus/all-3m-products/ /3M-Aluminum-Foil-Tape425/?N=5002385+3293241744\&rt=rud

[9] Sinske, J., Govers, Y., Jelicic, G. et al., "HALO flight test with instrumented under-wing stores for aeroelastic and load measurements in the DLR project iLOADS", CEAS Aeronaut J (2018) 9: 207. https://doi.org/10.1007/s13272-0180294-3 


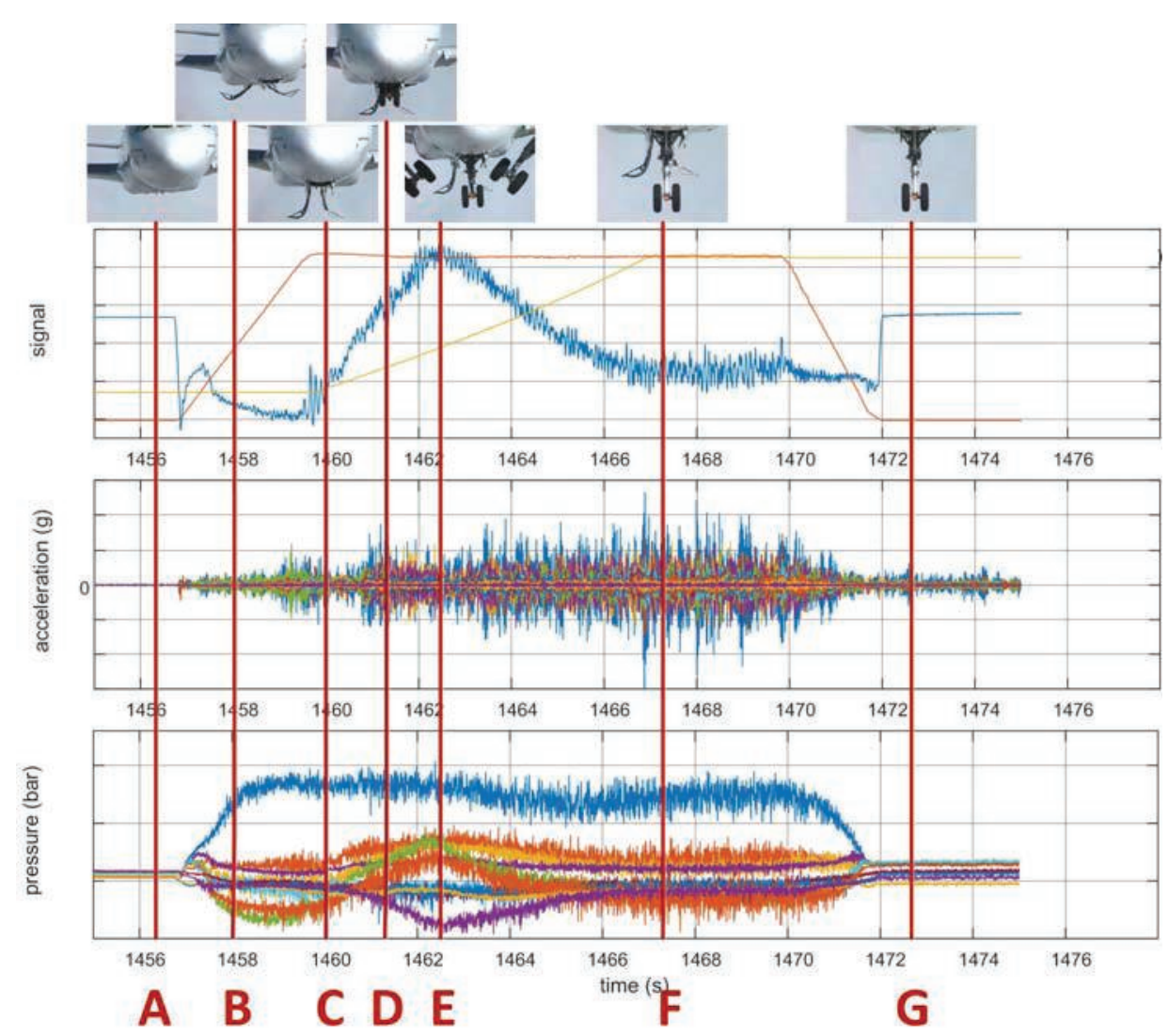

Figure 14. Deployment of LG, acceleration and pressures on left-hand MLG door at 250kts

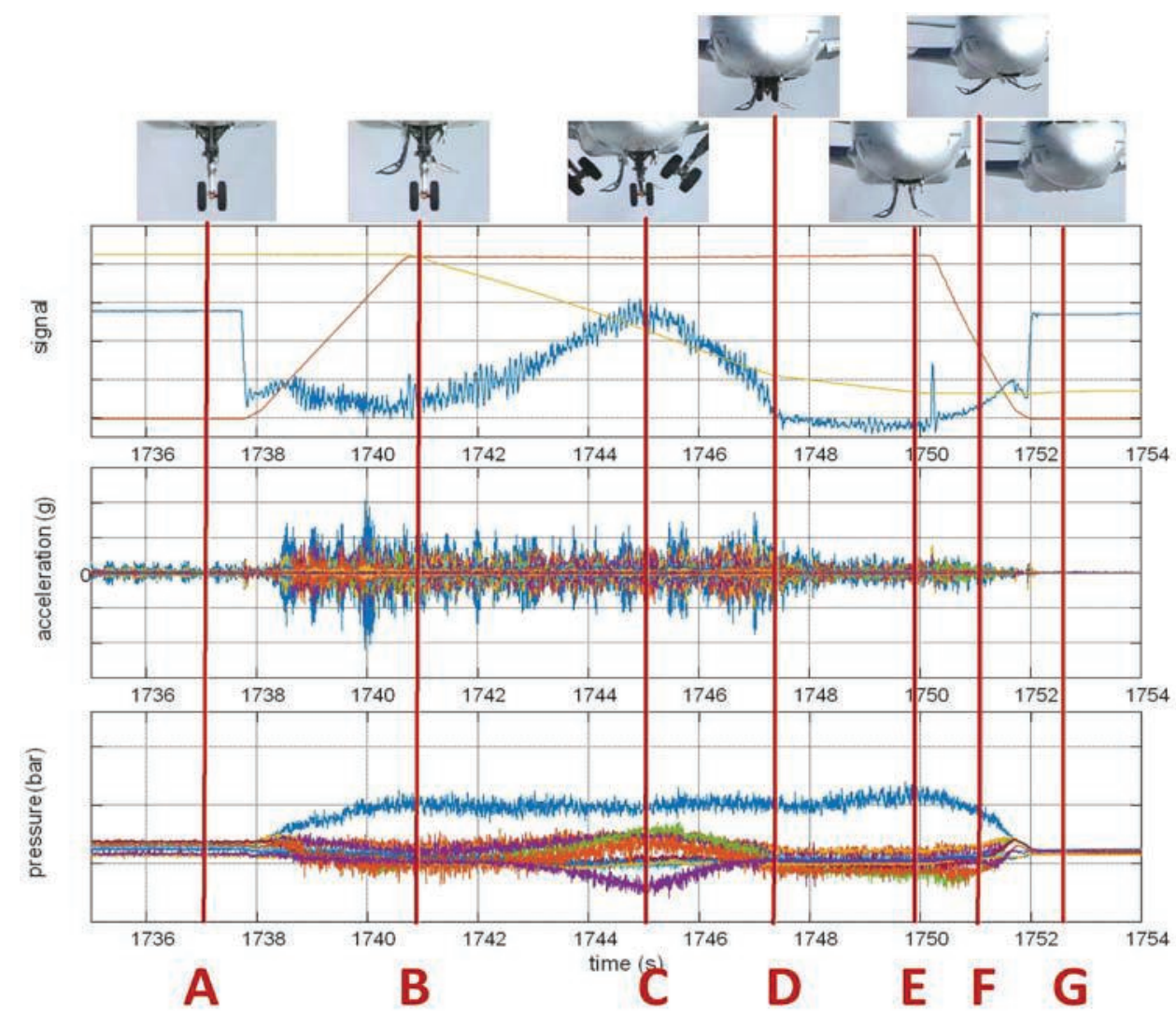

Figure 15. Retraction of LG, acceleration and pressures on left-hand MLG door at 220kts 